\title{
NOUVELle
}

\section{Une myosine non \\ conventionnelle contrôle la formation de l'axone}

Evelyne Coudrier ${ }^{1}$, Olga Iuliano ${ }^{2}$

\author{
${ }^{1}$ Institut Curie, PSL Research University, CNRS, UMR 144, \\ 26 rue d'Ulm, 75005 Paris, France. \\ 2Université Paul Sabatier UMR5169 (MINDING), \\ Toulouse, France. \\ coudrier@curie.fr
}

> Plusieurs myosines de type 1 possédant une seule chaîne lourde (myosines lb, lc et ld) ont été identifiées dans le cerveau $[1,2]$. Elles contribuent dans d'autres types cellulaires au trafic membranaire le long des voies d'endocytose et d'exocytose et participent à la formation de protrusions cellulaires, comme les filopodes ou les lamellipodes [3-5]. Étant donné leur interaction avec les filaments d'actine due à leur activité motrice, elles régulent également l'architecture des réseaux de filaments d'actine [3, 5]. De plus, elles interagissent directement avec les lipides membranaires via un motif homologue à la pleckstrine $(\mathrm{PH})$ dans leur domaine carboxy-terminal [3]. Couplant le cytosquelette d'actine aux membranes, elles peuvent contrôler la forme de ces dernières [5]. Nous avons étudié récemment le rôle d'une de ces myosines, la myosine lb (Myolb), dans le développement de neurones en culture primaire et en particulier, la formation de l'axone [6].

Les axones sont l'autoroute de l'information transportant toutes les données que nous utilisons pour détecter notre environnement et réagir en conséquence. Au cours du développement, différents types de neurones présentent dans un premier temps une morphologie multipolaire avec des protrusions, ou neurites, dynamiques de tailles

$(\rightarrow)$ Voir la Nouvelle de R. Chérau et al. $\mathrm{m} / \mathrm{s} \mathrm{n}^{\circ} 1$, janvier 2018, page 17 similaires [7] $(\rightarrow)$.

Puis, l'allongement rapide d'un neurite induit la polarisation du neurone et spécifie le site d'initiation de l'axone (Figure IA, ARNi contrôle) [8]. Nous avons observé que la réduction, par ARN interférent (ARNi), de l'expression de Myolb inhibe la formation de l'axone (Figure IA et IB), alors que la surproduction de la protéine augmente le nombre de protrusions apparentées à un axone [6]. Les filaments d'actine participent à la formation de l'axone à deux niveaux : les cônes de croissance, à l'extrémité aplatie des neurites (Figure IA GC), et la propagation le long des neurites de structures apparentées aux cônes de croissance, appelées vagues d'actine (Figure 2). Nous avons étudié l'impact de Myolb sur ces deux types de structures.

En accord avec les observations précédentes montrant que les cônes de croissance et les vagues d'actine sont des structures apparentées, nous avons observé en microscopie électronique que ces deux structures présentent une architectures des filaments d'actine similaires avec des réseaux branchés et des faisceaux $[6,9]$. Nos travaux indiquent également que Myolb est un constituant commun à ces deux structures. Myolb est présent dans les cônes de croissance et se propage le long des neurites avec l'actine. Comme rapporté pour la myosine 1 de l'amibe Dictyostelium, nous avons détecté une accumulation de Myolb endogène à l'avant de certaines vagues [10]. Après leur propagation vers l'extrémité du neurite, les vagues d'actine fusionnent avec les cônes de croissance et entraînent l'allongement du neurite [9]. Nous avons observé que la déplétion de Myolb inhibe la propagation antérograde des vagues d'actine. En contribuant à la direction de la propagation des vagues d'actine, Myolb participe par conséquent à l'élongation du neurite qui formera l'axone. Il a été récemment montré qu'en élargissant les neurites, les vagues d'actine augmentent la polymérisation des microtubules et en conséquence le transport de protéines déterminantes pour former l'axone à l'extrémité du neurite [11]. En accord avec cette étude, nous avons observé que l'inhibition de l'expression de Myolb perturbe les mouvements stochastiques de Kif5C, une kinésine qui transporte des protéines impliquées dans l'initiation de la formation de l'axone. À l'inverse l'augmentation de l'expression de Myolb accélère le déplacement de cette kinésine à l'extrémité des neurites. Ainsi, en régulant la propagation antérograde des vagues d'actine le long du neurite, Myolb favorise l'élongation des microtubules et l'acheminement des facteurs nécessaires à la formation de l'axone à son extrémité.

Le réseau d'actine impacte également la polymérisation des microtubules dans les cônes de croissance et en conséquence le transport de protéines impliquées dans la spécification des axones jusqu'à l'extrémité du neurite qui deviendra un axone $[11,12]$. Plus le réseau d'actine est stable et important moins les microtubules et le transport dépendant des microtubules peuvent atteindre l'extrémité des cônes de croissance. Nous avons observé que l'inhibition de l'expression de Myolb augmente la taille du réseau d'actine dans les cônes de croissance (FigurelA et 1C) alors que la surexpression de Myolb en restreint la taille [6]. En régulant la taille du réseau d'actine dans les cônes de croissance, Myolb peut donc aussi réguler 
A

\section{ARNi contrôle}
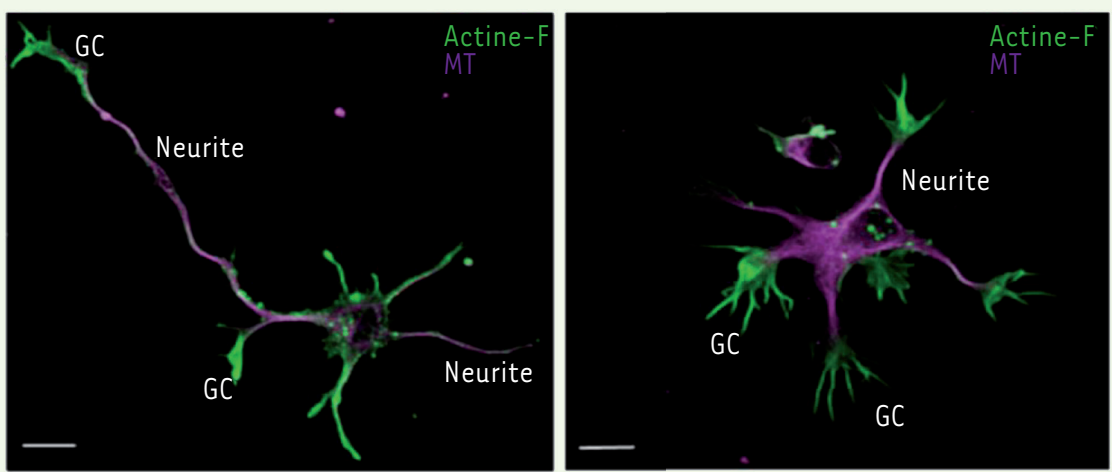

B

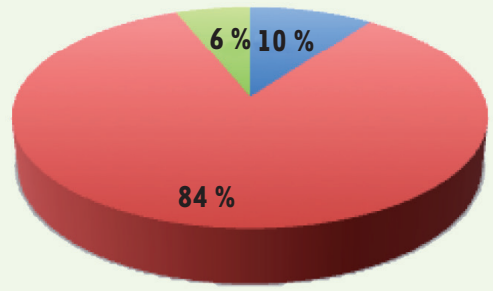

0 axone

1 axone

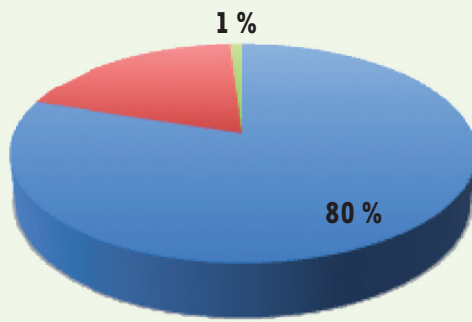

C

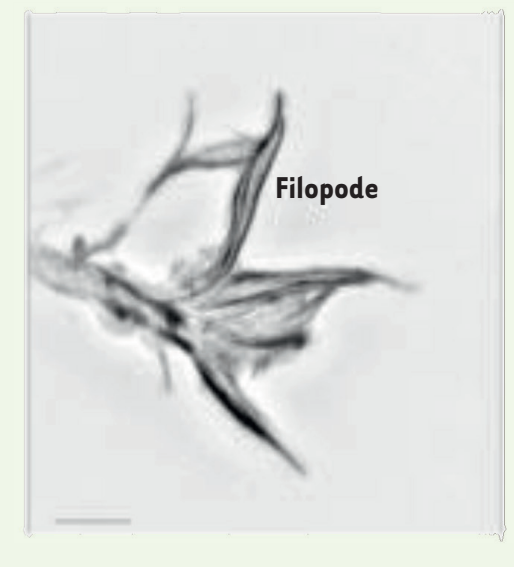

$>1$ axone

ARNi Myolb

Figure 1. Impact de Myolb sur les cônes de croissances et la formation des axones. A. Les cellules de culture primaire de neurones corticaux de souris ont été transfectées avec des ARNi contrôle ou ARNi dirigés contre Myolb et analysées par immunofluorescence pour la distribution des filaments d'actine (Actine-F) et des microtubules (MT), ce qui permet de visualiser les neurites et les cônes de croissance (GC). Après deux jours de culture, on note que le neurone transfecté avec les ARNi contrôle présente un neurite allongé alors que celui transfecté avec les ARNi Myolb présente des neurites de tailles similaires (barre $=5 \mu \mathrm{m})$. B. Pourcentage de neurones présentant 0 , un ou plusieurs axones après transfection d'ARNi contrôle ou ARNi Myolb après 4 jours de culture. Les axones sont identifiés à l'aide d'un anticorps dirigé contre la protéine Tau. $80 \%$ des neurones transfectés avec des ARNi Myolb ne présentent pas d'axone alors que $84 \%$ de ceux transfectés avec I'ARNi contrôle présentent un axone à ce stade. C. Images représentatives de la distribution des filaments d'actine dans des cônes de croissance de neurones transfectés avec des ARNi contrôle ou Myolb et analysées par microscopie super résolutive SIM (illumination structurée). On note une augmentation importante de la densité du réseau de filaments d'actine en absence de Myolb ainsi que des filopodes très courts en comparaison avec le cône de croissance d'un neurone contrôle (barre $=2 \mu \mathrm{m}$ ). la polymérisation des microtubules et le transport des protéines qui initient la formation de l'axone.

Ces résultats indiquent que Myolb contribue à la formation de l'axone en régulant l'architecture de l'actine dans les vagues et les cônes de croissance. Dans ces deux cas, une partie des filaments d'actine s'organisent en faisceaux pour former les filopodes, protrusions qui bordent le cône de croissance (Figure IC) et sont présentes à la surface des vagues d'actine ; ces protrusions déterminent la direction du mouvement des cônes de croissance et des vagues d'actine en explorant l'environnement. L'inhibition de l'expression de Myolb diminue la durée de vie des filopodes présents dans les vagues d'actine et les cônes de croissance, réduit leur longueur et réduit la densité de l'actine dans les filopodes des cônes de croissance (Figure 1C) [7]. La formation d'un filopode nécessite que les filaments d'actine organisés en faisceaux interagissent avec la membrane plasmique [13]. La manipulation d'un mutant pour l'interaction de Myolb avec les lipides membranaires ainsi que l'analyse d'un mutant dépourvu de son activité motrice ont montré que ces deux propriétés de Myolb sont nécessaires à la formation des axones. En liant mécaniquement les faisceaux d'actine à la membrane, Myolb peut stabiliser les filopodes à la fois dans les vagues d'actine et les cônes de croissance. 


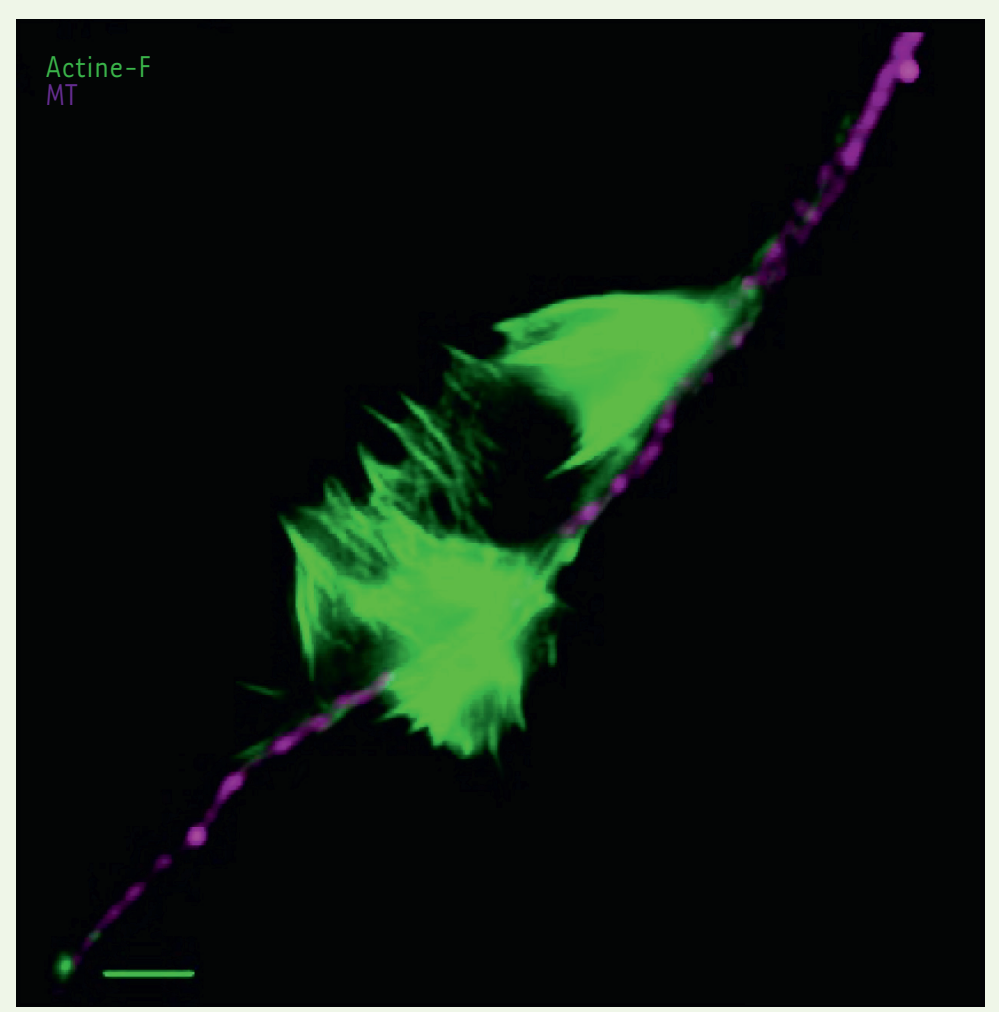

Figure 2. Une vague d'actine le long d'un neurite. Un neurite de neurone cortical de souris en culture primaire a été analysé par immunofluorescence et microscopie super résolutive SIM (illumination structurée) pour la distribution des filaments d'actine (Actine-F) et des microtubules (MT). On note un réseau d'actine formant une «vague d'actine » le long de ce neurite (barre $=2 \mu \mathrm{m}$ ).

En conclusion, nos données [7] démontrent que Myolb est nécessaire à la formation de l'axone dans des cultures primaires de neurones en contrôlant l'organisation de l'actine dans les vagues d'actine se propageant le long des neurites et dans les cônes de croissance à l'extrémité des neurites. Myolb régule notamment la formation des filopodes dans ces deux structures et affecte indirectement la polymérisation des microtubules et le transport des facteurs initiant la formation de l'axone. Le rôle de cette myosine pour la régénération des axones et les pathologies axonales reste à explorer. $\diamond$ An unconventional myosin controls the axon formation

\section{LIENS D'INTÉR}

Les auteurs déclarent n'avoir aucun lien d'intérêt concernant les données publiées dans cet article.

\section{RÉFÉRENCES}

1. Sherr EH, Joyce MP, Greene LA. Mammalian myosin I alpha, I beta, and I gamma: new widely expressed genes of the myosin I family. J Cell Biol 1993; 120 : 1405-16.

2. Lewis AK, Bridgman PC. Mammalian myosin I alpha is concentrated near the plasma membrane in nerve growth cones. Cell Motil Cytoskeleton 1996 ; 33 : 130-50.

3. McIntosh BB, Ostap EM. Myosin-I molecular motors at a glance. J Cell Sci 2016 ; 129 : 2689-95.

4. Prosperi MT, Lepine P, Dingli F, et al. Myosin $1 b$ functions as an effector of $\mathrm{EphB}$ signaling to control cell repulsion. J Cell Biol $2015 ; 210: 347-61$.

5. Almeida CG, Yamada A, Tenza D, et al. Myosin lb promotes the formation of post-Golgi carriers by regulating actin assembly and membrane remodelling at the trans-Golgi network. Nat Cell Biol $2011 ; 13$ : 779-89.

6. Iuliano 0, Yoshimura A, Prosperi MT, et al. Myosin lb promotes axon formation by regulating actin wave propagation and growth cone dynamics. J Cell Biol $2018 ; 217: 2033-46$.

7. Chéreau R, Angibaud J, Nägerl VU. La plasticité axonale révélée par imagerie super-résolution STED. Med Sci (Paris) 2018 ; 34 : 17-20.

8. Dotti CG, Sullivan CA, Banker GA. The establishment of polarity by hippocampal neurons in culture. J Neurosci $1988 ; 8: 1454-68$.

9. Flynn KC, Pak CW, Shaw AE, et al. Growth cone-like waves transport actin and promote axonogenesis and neurite branching. Dev Neurobiol 2009 ; 69 : 761-79.

10. Brzeska H, Pridham K, Chery G, et al. The association of myosin IB with actin waves in dictyostelium requires both the plasma membrane-binding site and actin-binding region in the myosin tail. PLoS One 2014 ; 9 : e94306.

12. Winans AM, Collins SR, Meyer T. Waves of actin and microtubule polymerization drive microtubule-based transport and neurite growth before single axon formation. Elife 2016 ; 5 .

13. Bradke F, Dotti CG. The role of local actin instability in axon formation. Science $1999 ; 283$ : 1931-4.

14. Nambiar R, McConnell RE, Tyska MJ. Myosin motor function: the ins and outs of actin-based membrane protrusions. Cell Mol Life Sci 2010 ; 67 : 1239-54.

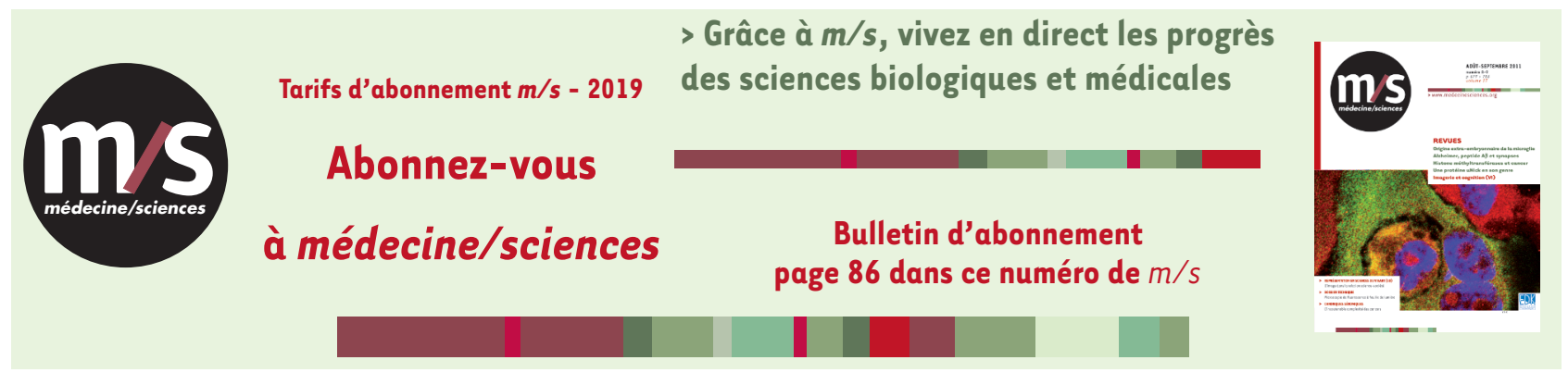

